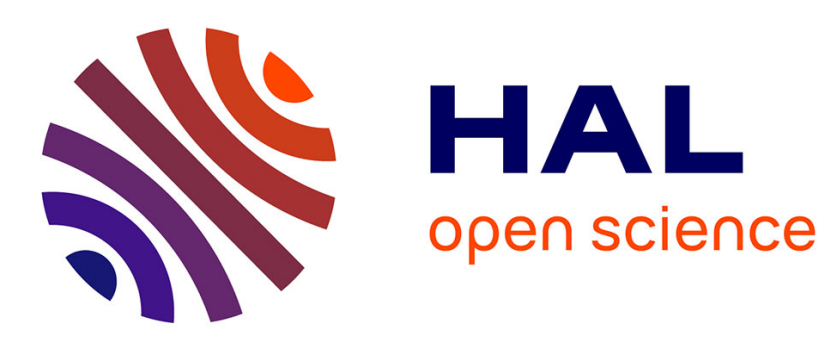

\title{
Structure cristalline de la L-leucyl-L-leucyl-L-tyrosine chlorhydraté
}

\author{
Jean Delettré, Jean Berthou, Alain Lifchitz, Pierre Jollès
}

\section{To cite this version:}

Jean Delettré, Jean Berthou, Alain Lifchitz, Pierre Jollès. Structure cristalline de la L-leucyl-L-leucylL-tyrosine chlorhydraté. Acta Crystallographica Section C : Structural Chemistry [2014-..], 1988, 44, pp.905-907. 10.1107/S0108270188000940 . hal-00015166

\section{HAL Id: hal-00015166 https://hal.science/hal-00015166}

Submitted on 24 Feb 2006

HAL is a multi-disciplinary open access archive for the deposit and dissemination of scientific research documents, whether they are published or not. The documents may come from teaching and research institutions in France or abroad, or from public or private research centers.
L'archive ouverte pluridisciplinaire HAL, est destinée au dépôt et à la diffusion de documents scientifiques de niveau recherche, publiés ou non, émanant des établissements d'enseignement et de recherche français ou étrangers, des laboratoires publics ou privés. 


\title{
Structure Cristalline de la L-Leucyl-L-leucyl-L-tyrosine Chlorhydraté
}

\author{
Par Jean Delettré, Jean Berthou et Alain Lifchitz \\ Laboratoire de Minéralogie et Cristallographie ( $L A C N R S n^{\circ}$ 9), Université Pierre et Marie Curie, \\ 75231 Paris CEDEX 05, France \\ et Pierre Jollès \\ Laboratoire des Protéines (UA CNRS $n^{\circ} 1188$ ), Université Paris V, 45 rue des Saints Pères, \\ 75270 Paris CEDEX 06, France
}

(Reçu le 23 septembre 1987, accepté le 20 janvier 1988)

\begin{abstract}
C}_{21} \mathrm{H}_{34} \mathrm{~N}_{3} \mathrm{O}_{5}^{+} \cdot \mathrm{Cl}^{-}, \quad M_{r}=444 \cdot 0, \quad$ orthorhombic, $P 22_{1} 2_{1}, a=26.074(5), b=17.591$ (4), $c$ $=5.224(2) \AA, \quad V=2396 \cdot 1(10) \AA^{3}, Z=4, \quad C u K \alpha$, $\lambda=1.5418 \AA, \mu=1.71 \mathrm{~mm}^{-1}, \quad F(000)=952, D_{x}=$ $1.231 \mathrm{Mg} \mathrm{m}^{-3}$, room temperature, final $R=0.080$ and $w R=0.070$ for 2439 reflections $\left[(\sin \theta) / \lambda>0.03 \AA^{-1}\right]$. The peptide groups are planar; torsion angles $(-101$ and $-88^{\circ}$ ) indicate a roughly helical structure. The peptide bonds have a trans conformation. The crystal structure is stabilized by a network of hydrogen bonds.
\end{abstract}

Introduction. Cette étude fait suite à celle du tripeptide L-glycyl-leucyl-phenylalanine (Delettré, Berthou, Lifchitz \& Jollès, 1988). Il a été montré (Jollès, Parker, Migliore-Samour, Alliel, Zerial \& Werner, 1982) que des fragments de la caséine humaine, une des fractions protéiques principales du lait maternel, possédaient des propriétés immuno-stimulantes. De cette caséine, de courts peptides actifs, ont pu être isolés et caractérisés comme l'hexapeptide Val-Glu-Pro-Ile-Pro-Tyr (Parker et al., 1984). Mieux encore, des tripeptides de même origine possèdent cette propriété (Berthou, MiglioreSamour, Lifchitz, Delettré, Floc'h \& Jollès, 1987).

C'est dans ce contexte que nous proposons l'étude du tripeptide naturel (LLT) afin de pouvour vérifier ultérieurement l'hypothèse d'une influence conformationnelle sur son activité.

Partie expérimentale. Ce peptide est peu soluble et cristallise mal. Une recherche systématique a été entreprise pour déterminer le meilleur solvant. Parmi les nombreux mélanges essayés une solution, acidifiée avec $\mathrm{HCl}(0,1 N)$, de DMA, $\mathrm{H}_{2} \mathrm{O}(1: 3)$ a été retenue et aprés évaporation lente a donné naissance à des cristaux de taille moyenne $(2,0 \times 0,2 \times 0,1 \mathrm{~mm})$, non hygroscopiques et stables à la température ambiante. Mesures: diffractomètre Philips, PW 1100, monochromateur graphite, température ambiante; mesure des intensites par 'flying stepscan'; 2945 réflexions mesurées; 2439 observées avec $I \geq 2 \sigma$; réflexions de 0108-2701/88/050905-03\$03.00
Tableau 1. Coordonnées atomiques fractionnaires $\left(\times 10^{4}\right)$ avec leurs écarts-type entre parenthèses et facteurs d'agitation thermique équivalents

\begin{tabular}{|c|c|c|c|c|}
\hline \multirow[b]{2}{*}{ Leucy] } & \multicolumn{3}{|c|}{$B_{\text {éq }}=\frac{4}{3} \sum_{i} \sum_{j} \beta_{i j} \mathbf{a}_{i} \mathbf{a}_{j}$} & \multirow[b]{2}{*}{$B_{\text {eq }}\left(\AA^{2}\right)$} \\
\hline & $x$ & $y$ & $z$ & \\
\hline $\begin{array}{l}\mathrm{N} \\
\mathrm{C} \alpha \\
\mathrm{C}^{\prime} \\
\mathrm{O} \\
\mathrm{C} \beta \\
\mathrm{C} \gamma \\
\mathrm{C} \delta \\
\mathrm{C} \delta(2)\end{array}$ & $\begin{array}{l}2232(2) \\
2336(2) \\
2695(2) \\
2569(2) \\
1832(2) \\
1872(3) \\
2103(3) \\
1351(3)\end{array}$ & $\begin{array}{l}4232(3) \\
3421(3) \\
3398(4) \\
3672(4) \\
3001(3) \\
2165(4) \\
1745(4) \\
1841(5)\end{array}$ & $\begin{array}{r}120(9) \\
-439(10) \\
-2749(10) \\
-4763(8) \\
-956(13) \\
-1420(14) \\
916(17) \\
-2089(22)\end{array}$ & $\begin{array}{l}4.8 \\
4.2 \\
4.7 \\
8.9 \\
5.3 \\
6.1 \\
8.2 \\
9.4\end{array}$ \\
\hline $\begin{array}{l}\text { Leucyl } \\
\mathrm{N} \\
\mathrm{C} \alpha \\
\mathrm{C}^{\prime} \\
\mathrm{O} \\
\mathrm{C} \beta \\
\mathrm{C} \gamma \\
\mathrm{C} \delta(1) \\
\mathrm{C} \delta(2)\end{array}$ & $\begin{array}{l}3144(2) \\
3533(2) \\
3529(2) \\
3508(2) \\
4060(2) \\
4505(2) \\
5012(4) \\
4539(6)\end{array}$ & $\begin{array}{l}3072(2) \\
3034(3) \\
2253(3) \\
2179(2) \\
3217(3) \\
3207(5) \\
3248(12) \\
3857(8)\end{array}$ & $\begin{array}{l}-2383(8) \\
-4359(10) \\
-5593(10) \\
-7964(7) \\
-3189(13) \\
-5121(18) \\
-3547(28) \\
-6422(29)\end{array}$ & $\begin{array}{r}4.0 \\
3.8 \\
3.6 \\
4.9 \\
4.9 \\
7.8 \\
20.4 \\
16,9\end{array}$ \\
\hline $\begin{array}{l}\text { Tyrosin } \\
\mathrm{N} \\
\mathrm{C} \alpha \\
\mathrm{C}^{\prime} \\
\mathrm{O}^{\prime} \\
\mathrm{O}^{\prime \prime} \\
\mathrm{C} \beta \\
\mathrm{C} \gamma \\
\mathrm{C} \delta(1) \\
\mathrm{C} \delta(2) \\
\mathrm{C} \varepsilon(1) \\
\mathrm{C} \varepsilon(2) \\
\mathrm{C} \zeta \\
\mathrm{O} \zeta\end{array}$ & $\begin{array}{l}3560(2) \\
3608(2) \\
3093(2) \\
3099(1) \\
2728(1) \\
3899(2) \\
4456(2) \\
4786(2) \\
4654(3) \\
5304(2) \\
5173(3) \\
5494(2) \\
5991(2)\end{array}$ & $\begin{array}{r}1655(2) \\
894(3) \\
543(3) \\
132(2) \\
614(2) \\
376(3) \\
573(3) \\
275(4) \\
1051(4) \\
459(4) \\
1210(5) \\
915(4) \\
1116(3)\end{array}$ & $\begin{array}{l}-4079(8) \\
-5030(11) \\
-5528(12) \\
-7665(9) \\
-4194(10) \\
-3136(12) \\
-2997(12) \\
-4810(14) \\
-1106(14) \\
-4773(16) \\
-1020(16) \\
-2871(17) \\
-2772(14)\end{array}$ & $\begin{array}{l}3.8 \\
3.6 \\
4.2 \\
5.8 \\
5.8 \\
4.7 \\
4.4 \\
5,6 \\
6,2 \\
6,3 \\
7.0 \\
6.3 \\
9.5\end{array}$ \\
\hline $\begin{array}{l}\text { Chlore } \\
\mathrm{Cl}\end{array}$ & $3329(1)$ & $4931(1)$ & $-9566(4)$ & 6.0 \\
\hline
\end{tabular}

contrôle: $231,12, \overline{1}, 0, \overline{2} \overline{3} \overline{1}$ variations maximales de $0,4 \%$ autour de la valeur moyenne. $0 \leq h \leq 32$, $0 \leq k \leq 22,0 \leq l \leq 6$; paramètres cristallins obtenus à partir de 25 réflexions $5<\theta<20^{\circ}$.

Corrections de Lorentz-polarisation, pas de correction d'absorption. $[(\sin \theta) / \lambda]_{\max }=0,609 \AA^{-1}$; résolution par méthodes directes: programme MULTAN avec utilisation de la partie YZARC (Main, (C) 1988 International Union of Crystallography 
Tableau 2. Longueurs de liaison $(\AA)$, angles de valence $\left(^{\circ}\right)$ et angles de torsion $\left({ }^{\circ}\right)$, les déviations standards étant données entre parenthèses

Angles de torsion en accord avec les normes de la IUPAC-IUB Commission on Biochemical Nomenclature (1970).

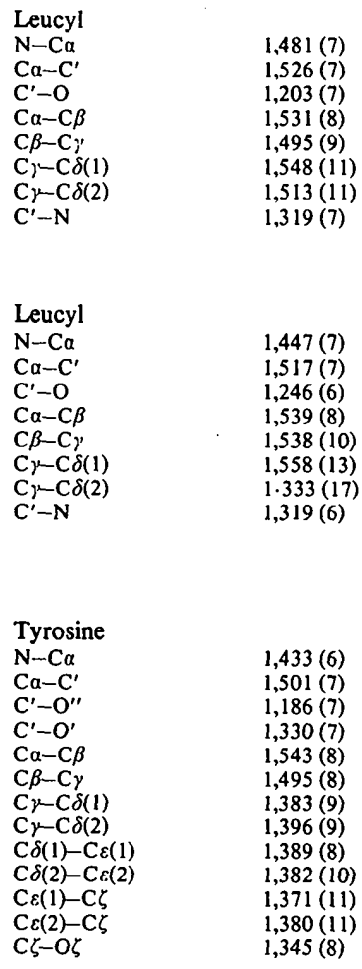

Angles dièdres du squelette $\left(^{\circ}\right)$ Leucyl $\begin{array}{rr}\psi & 119,6(5) \\ \omega & -177,4(5)\end{array}$

Leucyl

$$
\begin{array}{lr}
\varphi & -100,5(6) \\
\psi & -52,4(6) \\
\omega & -174,0(4)
\end{array}
$$

Tyrosine

$$
\varphi \quad-87,9(6)
$$

$\begin{array}{ll}\mathrm{N}-\mathrm{C} \alpha-\mathrm{C}^{\prime} & 107,1(5) \\ \mathrm{N}-\mathrm{C} \alpha-\mathrm{C} \beta & 110, \mathrm{I}(4) \\ \mathrm{C}-\mathrm{C} \alpha-\mathrm{C} \beta & 111,9(5) \\ \mathrm{C} \alpha-\mathrm{C} \beta-\mathrm{C} \gamma & 116,4(5) \\ \mathrm{C} \beta-\mathrm{C} \gamma-\mathrm{C} \delta(1) & 111,6(6) \\ \mathrm{C} \beta-\mathrm{C} \gamma-\mathrm{C} \delta(2) & 111,3(6) \\ \mathrm{C} \delta(1)-\mathrm{C} \gamma-\mathrm{C} \delta(2) & 110,6(7) \\ \mathrm{C} \alpha-\mathrm{C}^{\prime}-\mathrm{O} & 120,9(5) \\ \mathrm{C} \alpha-\mathrm{C}^{\prime}-\mathrm{N} & 116,2(5) \\ \mathrm{O}-\mathrm{C}^{\prime}-\mathrm{N} & 122,9(5)\end{array}$

$\begin{array}{ll}\mathrm{C}^{\prime}-\mathrm{N}-\mathrm{C} \alpha & 122,6(4) \\ \mathrm{N}-\mathrm{C} \alpha-\mathrm{C}^{\prime} & 109,9(4) \\ \mathrm{N}-\mathrm{C} \alpha-\mathrm{C} \beta & 109,4(4) \\ \mathrm{C}^{\prime}-\mathrm{C} \alpha-\mathrm{C} \beta & .111,3(4) \\ \mathrm{C} \alpha-\mathrm{C} \beta-\mathrm{C} \gamma & 114,3(6) \\ \mathrm{C} \beta-\mathrm{C} \gamma-\mathrm{C} \delta(1) & 107,(8) \\ \mathrm{C} \beta-\mathrm{C} \gamma-\mathrm{C} \delta(2) & 112,0(9) \\ \mathrm{C} \delta(1)-\mathrm{C} \gamma-\mathrm{C} \delta(2) & 100,0(11) \\ \mathrm{C} \alpha-\mathrm{C}^{\prime}-\mathrm{O} & 121,1(5) \\ \mathrm{C} \alpha-\mathrm{C}^{\prime}-\mathrm{N} & 117,9(5) \\ 0-\mathrm{C}^{\prime}-\mathrm{N} & 121,0(5)\end{array}$

$\begin{array}{ll}\mathrm{C}^{\prime}-\mathrm{N}-\mathrm{C} \alpha & 122,9(4) \\ \mathrm{N}-\mathrm{C} \alpha-\mathrm{C}^{\prime} & 111,4(4) \\ \mathrm{N}-\mathrm{C} \alpha-\mathrm{C} \beta & 111,8(4) \\ \mathrm{C}^{\prime}-\mathrm{C} \alpha-\mathrm{C} \beta & 107,9(4) \\ \mathrm{C} \alpha-\mathrm{C} \beta-\mathrm{C} \gamma & 111,8(5) \\ \mathrm{C} \delta(1)-\mathrm{C} \gamma-\mathrm{C} \beta & 118,9(5) \\ \mathrm{C} \delta(2)-\mathrm{C} \gamma-\mathrm{C} \beta & 122,2(5) \\ \mathrm{C} \delta(1)-\mathrm{C} \gamma-\mathrm{C} \delta(2) & 118,9(5) \\ \mathrm{C} \gamma-\mathrm{C} \delta(1)-\mathrm{C} \varepsilon(1) & 120,4(6) \\ \mathrm{C} \gamma-\mathrm{C} \delta(2)-\mathrm{C} \varepsilon(2) & 120,5(6) \\ \mathrm{C} \delta(1)-\mathrm{C} \varepsilon(1)-\mathrm{C} \zeta & 119,9(7) \\ \mathrm{C} \delta(2)-\mathrm{C} \varepsilon(2)-\mathrm{C} \zeta & 119,7(7) \\ \mathrm{C} \varepsilon(1)-\mathrm{C} \zeta-\mathrm{C} \varepsilon(2) & 120,5(6) \\ \mathrm{C}(1)-\mathrm{C} \zeta-\mathrm{O} \zeta & 122,0(7) \\ \mathrm{C} \varepsilon(2)-\mathrm{C} \zeta-\mathrm{O} \zeta & 117,4(7) \\ \mathrm{O}^{\prime}-\mathrm{C}^{\prime}-\mathrm{C} \alpha & 111,0(5) \\ \mathrm{O}^{\prime \prime}-\mathrm{C}^{\prime}-\mathrm{C} \alpha & 125,0(5) \\ \mathrm{O}^{\prime \prime}-\mathrm{C}^{\prime}-\mathrm{O}^{\prime} & 124,0(5) \\ & \end{array}$

Angles dièdres des chaînes latérales $\left(^{\circ}\right)$ $\mathrm{N}-\mathrm{C} \alpha-\mathrm{C} \beta-\mathrm{C} \gamma \quad-177,0(5)$ $\begin{array}{lr}\mathrm{C} \alpha-\mathrm{C} \beta-\mathrm{C} \gamma-\mathrm{C} \delta(1) & 60,8(8) \\ \mathrm{C} \alpha-\mathrm{C} \beta-\mathrm{C} \gamma-\mathrm{C} \delta(2) & -175,9(7)\end{array}$

$\mathrm{N}-\mathrm{C} \alpha-\mathrm{C} \beta-\mathrm{C} \gamma$ $\begin{array}{rr}\mathrm{C} \alpha-\mathrm{C} \beta-\mathrm{C} \gamma-\mathrm{C} \delta(1) & -169,4(9) \\ \mathrm{C} \alpha-\mathrm{C} \beta-\mathrm{C} \gamma-\mathrm{C} \delta(2) & 81,9(10)\end{array}$

$\mathrm{N}-\mathrm{C} \alpha-\mathrm{C} \beta-\mathrm{C} \gamma$

$\mathrm{C} \alpha-\mathrm{C} \beta-\mathrm{C} \gamma-\mathrm{C} \delta(1)$

$\mathrm{C} \alpha-\mathrm{C} \beta-\mathrm{C} \gamma-\mathrm{C} \delta(2)$

$\mathrm{C} \beta-\mathrm{C} \gamma-\mathrm{C} \delta(1)-\mathrm{C} \varepsilon(1)$

$\mathrm{C} \beta-\mathrm{C} \gamma-\mathrm{C} \delta(2)-\mathrm{C} \varepsilon(2)$

$\mathrm{C} \gamma-\mathrm{C} \delta(1)-\mathrm{C} \varepsilon(1)-\mathrm{C} \zeta$

$\mathrm{C} \gamma-\mathrm{C} \delta(2)-\mathrm{C} \varepsilon(2)-\mathrm{C} \zeta$

$\mathrm{C} \delta(1)-\mathrm{C} \varepsilon(1)-\mathrm{C} \zeta-\mathrm{C} \varepsilon(2)$

$\mathrm{C} \delta(2)-\mathrm{C} \varepsilon(2)-\mathrm{C} \zeta-\mathrm{C} \varepsilon(1)$

$\mathrm{C} \delta(1)-\mathrm{C} \varepsilon(1)-\mathrm{C} \zeta-\mathrm{O} \zeta$

$\mathrm{C} \delta(2)-\mathrm{C} \varepsilon(2)-\mathrm{C} \zeta-\mathrm{O} \zeta$ du facteur $R$; minimisation de $\sum w\left(\left|F_{o}\right|-\left|F_{c}\right|\right)^{2}$ avec $w=1 / \sigma^{2}$; atomes $\mathrm{H}$ placés sur section de Fourierdifférence et affinés, sauf en ce qui concerne les méthyl $\mathrm{du}$ résidu Leu(2) (position théorique); coefficients d'agitation thermique anisotrope pour tous les atomes sauf $\mathrm{H}$; résidu de densité électronique sur sections de Fourier-différence finales 0,65 e $\AA^{-3} ;(\Delta / \sigma)_{\max }$ pour les $x, y, z$ des C,O: 0,1 . L'ion $\mathrm{Cl}^{-}$a été découvert et placé sur section de Fourier-différence.

Discussion. Les coordonnées atomiques sont reportées dans le Tableau $1,{ }^{*}$ les longueurs de liaison, angles de valence et angles de torsion dans le Tableau 2. La Fig. 1 montre la projection selon l'axe $c$.

Nous sommes en présence d'un chlorhydrate $\left(\mathrm{NH}_{3}^{+}\right.$, $\mathrm{Cl}^{-}$) tout à fait caractéristique et comparable à ce qui a pu être observé par exemple dans la cas du chlorhydrate de glycyl-glycine (Parthasarathy, 1969). Les liaisons peptidiques sont en conformation trans, les groupes peptidiques sont plans $\left(\omega=177\right.$ et $\left.174^{\circ}\right)$. Les angles de torsion montrent l'ébauche d'une structure hélicoïdale régulière $\left(\varphi=-101\right.$ et $\left.-88^{\circ}\right)$, sa conformation diffère donc de celle observée pour LGly-Leu-Phe.

Il faut noter la grande délocalisation de la chaîne terminale de la deuxième leucine, ce fait n'est pas rare, citons par exemple (Ishida et al., 1984).

* Les listes des facteurs de structure, des paramètres d'agitation thermique anisotrope, des angles de valence, et des coordonnées des atomes d'hydrogène, ont été déposées au dépôt d'archives de la British Library Document Supply Centre (Supplementary Publication No. SUP 44712: 20 pp.). On peut en obtenir des copies en s'adressant à: The Executive Secretary, International Union of Crystallography, 5 Abbey Square, Chester $\mathrm{CH} 1$ 2HU, Angleterre.

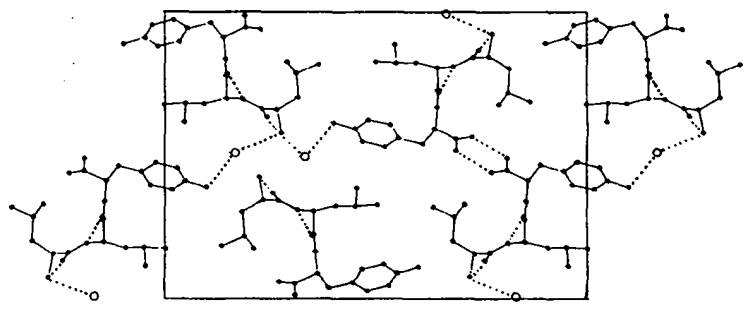

Fig. 1. Projection de l'arrangement moléculaire selon l'axe $c$. Les liaisons hydrogène sont représentées en pointillés.

Tableau 3. Liaisons hydrogène $(\AA)$

Fiske, Hull, Lessinger, Germain, Declercq \& Woolfson, 1980), facteurs de diffusion atomique (Cromer \& Mann, 1968). Affinement en matrice complète: programme AFFINE (Delettré, Mornon \& Lepicard, 1980) converge jusqu'à $R=0,080, w R=0,070$; la mauvaise qualité cristalline explique la valeur relativement élevée
$\mathrm{N}($ Leu 1) (i) $\mathrm{H} \cdots \mathrm{O}$ (Leu l) (ii)

$\mathrm{N}\left(\right.$ Leu 1) (i) $\mathrm{H} \cdots \mathrm{Cl}^{-}$(ii)

$\mathrm{N}($ Leu 1$)$ (i) $\mathrm{H} . . . \mathrm{Cl}^{-}$(iv)

$\mathrm{N}$ (Leu 2) (i) $\mathrm{H}$ … O (Leu 2) (ii)

$\mathrm{O} \zeta(\mathrm{i}) \mathrm{H} \cdot . \mathrm{Cl}^{-}$(iii)

$\mathrm{O}^{\prime}(\mathrm{i}) \mathrm{H} \cdots \mathrm{O}^{\prime \prime}(\mathrm{v})$

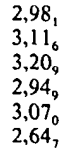

Les opérations de symétrie sont: (i) $x, y, z$; (ii) $x, y, 1+z$; (iii) $1-x,-\frac{1}{2}+y$, $-\frac{3}{2}-z$; (iv) $\frac{1}{2}-x, 1-y, \frac{1}{2}+z$; (v) $\frac{1}{2}-x,-y,-\frac{1}{2}+z$. 


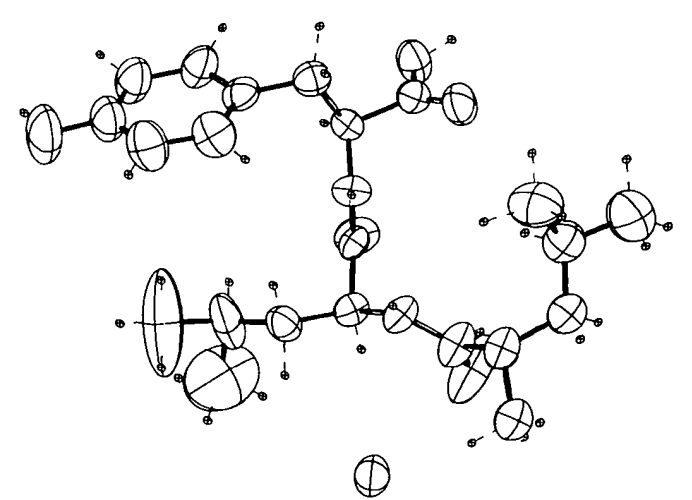

Fig. 2. Représentation des ellipsoïdes d'agitation thermique à $50 \%$ de probabilité.

L'édifice cristallin est stabilisé par un réseau de liaisons hydrogène donné par le Tableau 3. Il faut noter que les atomes $\mathrm{O}$ (Gly) et $\mathrm{N}$ (Phe) ne sont pas impliqués dans le réseau de liaisons hydrogène.

La Fig. 2 représente les ellipsoïdes d'agitation thermique.

\section{Références}

Berthou, J., Migliore-Samour, D., Lifchitz, A., Delettré, J., FloC'H, F. \& Jollés, P. (1987). Febs Lett. 218, 55-58.

Cromer, D. T. \& MANN, J. B. (1968). Acta Cryst. A24, 321-324.

Delettré, J., Berthou, J., Lifchitz, A. \& Jollès, P. (1988), Acta Cryst. C44, 902-904.

Delettré, J., Mornon, J. P. \& Lepicard, G. (1980). Acta Cryst. B36, 1430-1435.

Ishida, T., Kenmotsu, M., Mino, Y., Inoue, M., Fujiwara, T., Tomita, K., Kimura, T. \& SaKakibara, S. (1984). J. Biochem. 218, 677-689.

IUPAC-IUB COMmission ON BIochemical Nomenclature (1970). Biochemistry, 9, 3471-3479.

Jollès, P., Parker, F., Migliore-Samour, D., Alliel, P., ZERIAL, A. \& WERNER, G. H. (1982). J. Immunopharmacol. 4, 363-369.

Main, P., Fiske, S. J., Hull, S. E., Lessinger, L., Germain, G., DeClercQ, J.-P. \& Woolfson, M. M. (1980). MULTAN80. A System of Computer Programs for the Automatic Solution of Crystal Structures from X-ray Diffraction Data. Univ. de York, Angleterre, et de Louvain, Belgique.

Parker, F., Migliore-Samour, D., Floc'h, F., Zerial, A., Werner, G. H., Jollès, J., Casaretto, M., ZahN, H. \& Jollès, P. (1984). Eur. J. Biochem. 145, 677-682.

Parthasarathy, R. (1969). Acta Cryst. B25, 509-518. 